\title{
Ultrasound Can Accurately Diagnose Fibroadenomas in Women Less Than 35 Years
}

\author{
Mustafa Khanbhai*, Rita Borgen and Richard Dobrashian
}

Breast Unit, Burnley General Hospital, Burnley, UK

*Corresponding author: Mustafa Khanbhai, Breast Unit, Burnley General Hospital, East Lancashire NHS Trust, Casterton Avenue, Burnley, BB10 2PQ, UK, E-mail: mustafa.khanbhai@doctors.org.uk

\begin{abstract}
Introduction: Best practice guidelines suggest that patients $<25$ years with ultrasound diagnosis of fibroadenoma, biopsy can be avoided if certain radiological criteria are satisfied. There is a cohort of patients $<35$ years with ultrasound features suggestive of fibroadenoma who are being exposed to unnecessary biopsies. The aim of this study is to determine how many patients with ultrasound features of fibroadenoma have had normal histology and therefore could potentially avoid a biopsy.
\end{abstract}

Methods: Records of women presenting to East Lancashire NHS Trust Breast Unit between 2013 and 2015 were obtained. Only patients between 25 and 35 years at the age of presentation, with clinical benign breast lump and an USS diagnosis of fibroadenoma were included. Corresponding Fine Needle Aspiration (FNA) cytology was noted. Cost of FNA and specimen preparation was recorded.

Results: A total of 135 USS diagnosis of fibroadenomas were reviewed, of which there were 121 patients with a mean age of 28.9 years. $100 / 135(74.1 \%)$ of fibroadenomas were subjected to FNA, of which $75 / 135(55.6 \%)$ revealed cytology of C2, 22/135 (16.3\%) were C1. Only $1 / 135(0.7 \%)$ fibroadenoma was $\mathrm{C} 3$, which on subsequent core-biopsy was B2. There was $35 / 135(25.9 \%)$ fibroadenomas that were not subjected to FNA with a median age was 25 years. Equipment for FNA cost $£ 0.73$ and cytology was $£ 90$ for each specimen processed (total £9073).

Conclusion: This study has demonstrated that it is safe to increase the age of patients with presumed fibroadenoma from 25 to 35 years without the need to confirm the diagnosis with FNA. In our study, no cancers would have been missed in this age group providing strict sonographic and clinical criteria are used.

\section{Introduction}

Ultrasound (US) is a well-established technique in diagnostic imaging of the breast. As technology has im- proved so has the quality of US images. Therefore, the role of ultrasound has expanded beyond characterizing solid from cystic masses, but now also has a role in distinguishing between malignant and benign breast lumps such as fibroadenomas. Many studies have shown that FNA or core biopsy are not required to confirm the diagnosis of fibroadenomas in young women $[1,2]$ providing strict criteria are used; no known risk factors for breast malignancy; mass not rapidly enlarging; smooth discrete mobile mass on clinical examination, or lesion impalpable; well-defined homogeneously isoechoic or mildly hypoechoic solid mass; less than $3 \mathrm{~cm}$ in greatest dimension; ovoid shape, aligned parallel to the skin surface; smooth or gently lobulated contour (two or three lobulations only; no microlobulation); thin echogenic pseudocapsule; no calcification; no acoustic shadowing [1-3]. We propose that the age can be increased to 35 years providing the above strict criteria are used, and there is no clinical suspicion of a malignant lesion. The purpose of this study was to review the need to biopsy fibroadenomas in the 25 to 35 -year age group.

\section{Methods}

Records of women presenting to East Lancashire NHS Trust Breast Unit between 2013 and 2015 were obtained. Only patients between 25 and 35 years at the age of presentation, with clinical benign (P2-benign) breast lump and an USS diagnosis of fibroadenoma (U2-benign) were included in the study. All ultrasounds were performed by Breast Radiologists or Advanced Mammography Practitioners using an Aplio 500 ultrasound system (Toshiba), linear-array transducer with frequency range of 7 to $18 \mathrm{MHz}$ Corresponding Fine Needle Aspiration (FNA) cytology was noted and if there were

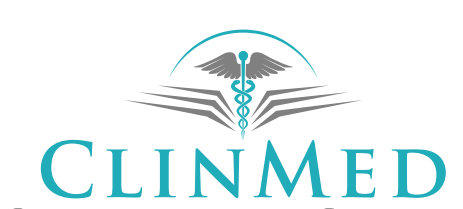

INTERNATIONAL LIBRARY
Citation: Khanbhai M, Borgen R, Dobrashian R (2017) Ultrasound Can Accurately Diagnose Fibroadenomas in Women Less Than 35 Years. Int J Radiol Imaging Technol 3:026. doi.org/10.23937/2572-3235.1510026 Received: May 04, 2017: Accepted: August 30, 2017: Published: September 01, 2017

Copyright: (C) 2017 Khanbhai M, et al. This is an open-access article distributed under the terms of the Creative Commons Attribution License, which permits unrestricted use, distribution, and reproduction in any medium, provided the original author and source are credited. 
any discrepancies, further information was sought. All cytology was classified $\mathrm{C} 1$ to $\mathrm{C} 5$ where $\mathrm{C} 1$, inadequate sample for testing; $\mathrm{C2}$, benign epithelial cells; $\mathrm{C} 3$, atypia more likely to be benign; $\mathrm{C} 4$, highly suspicious of malignancy; $C 4$, malignant present. A cytology report other than C2-benign was further assessed with core needle biopsy. Cost of FNA and cytology (specimen preparation) was recorded.

\section{Results}

A total of 135 USS diagnoses of fibroadenomas (U2) were reviewed, of which there were 121 patients with a mean age of 28.9 years. $100 / 135$ (74.1\%) of fibroadenomas were subjected to FNA, of which $75 / 135$ (55.6\%) revealed cytology of $\mathrm{C} 2,22 / 135$ (16.3\%) were $\mathrm{C} 1$ of which 4 were subjected to core-biopsy (1/4 B1, 2/4 B2, 1/4 B3) based on clinical judgement (Figure 1 ), where $B 1$, normal breast tissue; $B 2$, benign; $B 3$, benign but of uncertain malignancy potential; $B 4$, suspicious of malignancy; B5, malignant. The B3 lesion was surgically excised, and final histology revealed features of a fibroadenoma with no malignancy. Only $1 / 135(0.7 \%)$ fibroadenoma yielded a C3 diagnosis at FNA, which on subsequent core-biopsy was B2. 35/135 (25.9\%) fibroadenomas were not subjected to FNA, as 8 were known fibroadenomas, 5 had breast implants, 4 had previous excisions confirming fibroadenomas, 2 declined FNA and 2 had previous C2 on FNA. Of the remaining 14 fibroadenomas that did not have a FNA, the median age was $25.2 / 135$ (1.5\%) were excised and histology showed fibroadenoma. No patients had any immediate complications. Equipment cost of FNA is $£ 0.73$ and cytology $f 90$ for each specimen processed, the labour cost is not accounted for.

\section{Discussion}

Fibroadenomas are the most common surgically

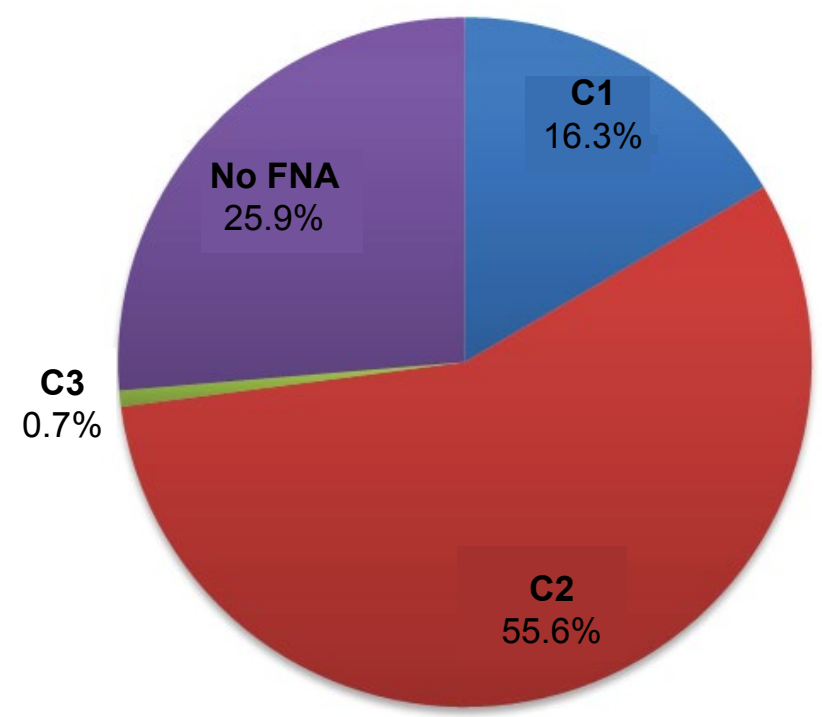

Figure 1: Outcome of all fibroadenomas $(n=135)$ and cytology of those subjected to FNA, fine needle aspiration, C1: Inadequate sample for testing; C2: Benign epithelial cells; C3: Atypia more likely to be benign. related breast masses in adolescents, accounting for $44-94 \%$ of biopsied breast lesions [3]. It has a peak incidence between 15 and 35 years and occurs in 25\% of asymptomatic women. Commonly regarded as a benign tumour of the breast, fibroadenoma represent a group of hyperplastic breast lobules called "aberrations of normal development and involution [4]", which is hormone dependent, which grows during pregnancy and involutes along with the rest of the breast in perimenopausal period [5]. Fibroadenomas typically present as firm, non-tender, discrete masses usually $2-3 \mathrm{~cm}$ in size, but can also be $<1 \mathrm{~cm}$ to greater that $10 \mathrm{~cm}$ in size. They are most commonly found in the upper outer quadrant of the breast. These benign masses enlarge slowly usually without associated pain or nipple and skin changes, but fluctuations in size may occur with the menstrual cycle; when symptoms are present, they last an average of 5 months [6]. In histological terms; macroscopically, a fibroadenomas are well-circumscribed and firm and the cut surfaces appear lobulated and bulging. Microscopically, fibroadenomas consist of a proliferation of epithelial elements arising from the terminal duct-lobular unit, and mesenchymal elements, though there are several histological variants [7]. The stroma proliferates

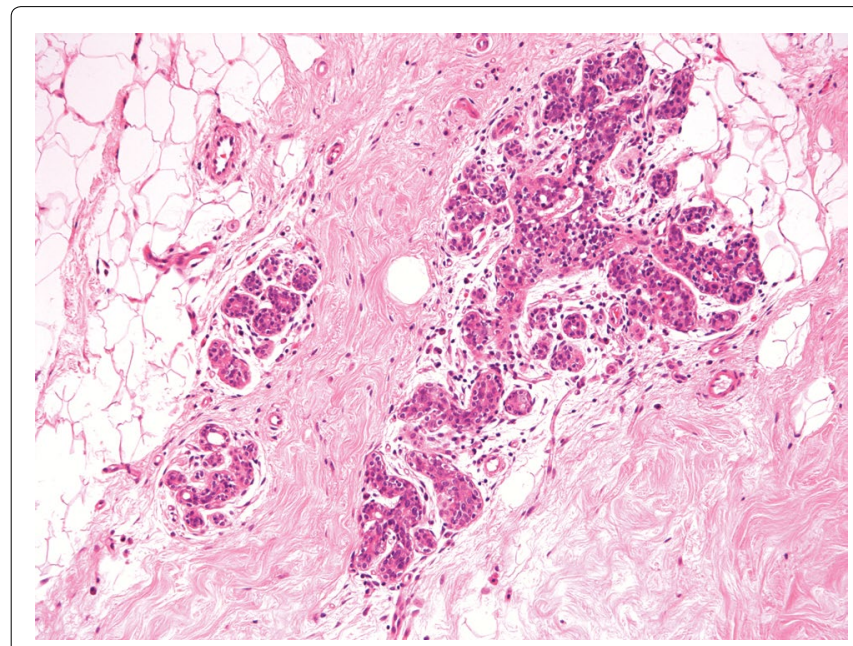

Figure 2: Microscopic appearance of normal breast tissue.

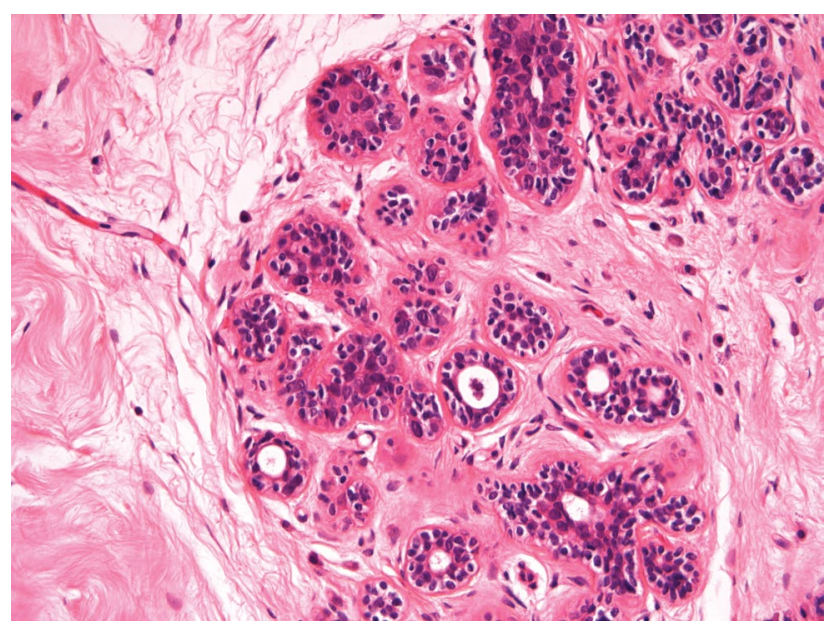

Figure 3: Microscopic appearance of normal breast tissue. 
around the tubular glands (pericanalicular growth) or compressed cleft-like ducts (intracanalicular growth) (Figure 2, Figure 3, Figure 4 and Figure 5).

Management of fibroadenomas usually follows a conservative approach as the malignant potential is extremely low. Currently in the UK, once an ultrasound diagnosis of fibroadenoma is made in patients greater than 25 years of age, they routinely undergo histological sampling as per the Association of Breast Surgery. However, in patients less than 25 years a biopsy need not be performed if the following criteria are satisfied: ultrasound reveals a solid lesion which has benign ultrasound features (e.g. ellipsoid shape [wider than tall]), a well-defined outline with smooth edges or fewer than four gentle lobulations. Ultrasound accuracy of breast lesions continues to improve resulting in reduction in histological sampling. The negative predictive value for a sonographic benign classification is $99.5 \%$ when strict criteria are used [8]. There are criteria as proposed by Maxwell, et al. [9] where biopsy of breast masses can be avoided. This includes; age less than 25 years; no known risk factors for breast cancer; mass not increasing significantly in size; clinical findings of a smooth dis-

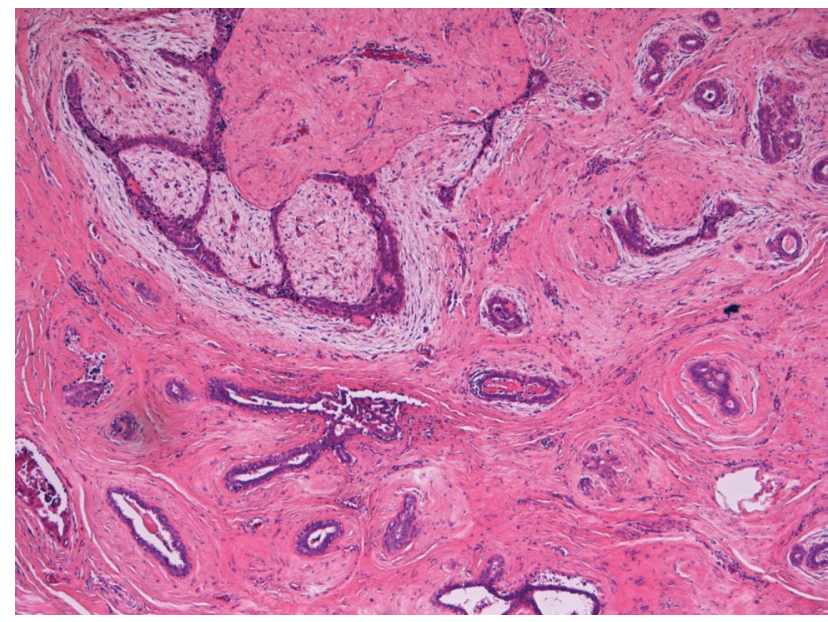

Figure 4: Microscopic appearance of fibroadenoma (high power).

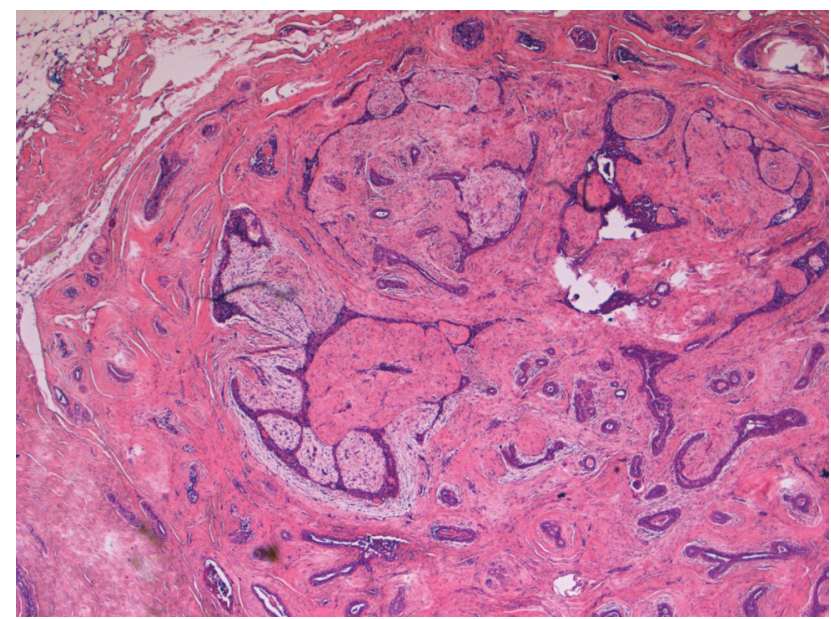

Figure 5: Microscopic appearance of fibroadenoma (low power). crete mobile lump or lump is impalpable; ultrasound reveals: well-defined homogeneously isoechoic or mildly hypoechoic solid lump; maximum dimension less than $30 \mathrm{~mm}$; ovoid shape, lying parallel to the surface of the skin; smooth or gently lobulated contour; thin echogenic pseudocapsule; lack of calcification; lack of acoustic shadowing (Figure 6 and Figure 7). Similar conclusions have been drawn from other studies [1-3]. If all these criteria are not met then the lesion should be classified as indeterminate and an appropriate biopsy performed. The breast unit in our hospital has dedicated breast radiologists who are more able to make an accurate judgement regarding the characteristics of a breast mass on ultrasound based on the above principles. Several authors have opted for conservative management of fibroadenoma below a certain age; 25 years [10], 35 years [11] and 40 years [12] have been suggested as age thresholds. We found that biopsies in patients up to 35 years of age with US features suggestive of fibroadenoma may not be not required when the criteria was met. In addition, no malignancies were missed in this age group.

Fibroadenoma resection specimen histology from

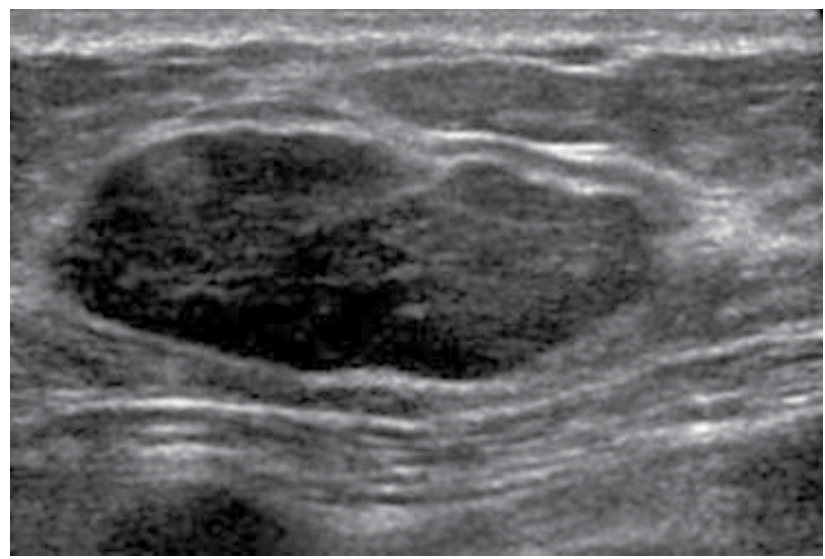

Figure 6: Ultrasound image showing a gently lobulated, oval shaped, well defined homogeneous mildly hypoechoic solid mass, less than $30 \mathrm{~mm}$ in maximum dimensions with a thin, echogenic pseudocapsule and showing no internal calcification or distal acoustic shadowing.

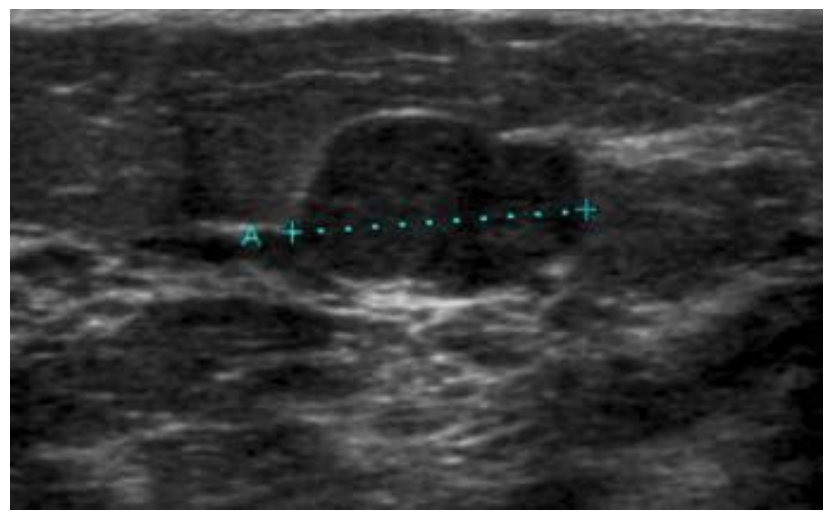

Figure 7: Ultrasound image showing a gently lobulated, oval shaped, well defined homogeneous mildly hypoechoic solid mass, less than $30 \mathrm{~mm}$ in maximum dimensions with a thin, echogenic pseudocapsule and showing no internal calcification or distal acoustic shadowing. 
previous literature suggests risk of malignancy around fibroadenomas occur frequently and mostly above the age of 35 years [7]. Kuijper A, et al. [7], documented a $2 \%$ for carcinoma-in situ within fibroadenoma, the youngest being 40 years of age. No invasive carcinoma was present within the 396 cases of fibroadenomas. Ozello, et al. [13], found an incidence of $0.3 \%$ for invasive and in situ carcinoma taken together and Deschenes, et al. [14], found one carcinoma in situ and one invasive carcinoma in 70 fibroadenomas (1.4\% each). Therefore the malignant potential of fibroadenomas in patients of a certain age bracket is very low. Even in terms of diagnosis in patients < 35-years-old, breast cancer rates are low compared to patients $>55$ years of age. Cancer Research UK documents the average number of new cases per year and age specific incidence rates per 100,000 females in the UK between 2010-2012 is 190 and 8.8 respectively for ages 25 to 29 years, with 548 and 26.4 respectively for ages 30 to 34 years [15].

Patients undergoing breast biopsy can experience clinically marked levels of anxiety, whether it is an open biopsy or needle biopsy. In fact, the anxiety levels for a breast biopsy have been reported to exceed the anxiety levels for patients who undergo elective surgery [16]. Clinically significant levels of anxiety have been reported in a sample of women who were anticipating, undergoing, and remembering breast needle biopsy [9]. Anxiety can also be caused by having to wait (usually a week, in our department) for histology results and then waiting for a follow-up appointment for the results to be relayed to the patient. Furthermore, the equipment cost of performing a FNA in 100 patients was $£ 9073$ without the added cost of personnel involved in processing and analysing the specimens. All FNAs were then discussed in the multidisciplinary meeting, and all patients were then seen in the outpatient clinic, which often have to be overbooked. Eliminating FNA in such patients would not only result in an increase in efficiency and cost-effectiveness, but also reduce the added anxiety that the procedure can cause to the patient.

There are a number of limitations in this study which must be taken into consideration. We did not evaluate other centres regionally treating breast disease. A multi-centre approach would not only give us more patient numbers, but also increase the validity of our proposal. We currently do not have a standard follow-up recommendation in patients less than 35 years with ultrasound diagnosis of fibroadenoma who have not undergone biopsy. We are however, in the process of developing local guidelines to manage patients less than 35 years presenting with suspected fibroadenoma.

\section{Conclusion}

Our study demonstrates that no malignancy was detected in the 135 patients below 35 years using the already established sonographic criteria for fibroadenoma. However, it is important to counsel patients who present with fibroadenoma and/or benign lump to seek prompt referral for further assessment should they notice any increase in size of other change in the mass. We propose, it is safe to extend the current guidelines of not needing to biopsy presumed fibroadenoma in women up to the age of 35 providing the strict sonographic criteria outlined above are used and there is no clinical suspicion of malignancy.

The authors declare that there is no conflict of interest regarding the publication of this paper.

\section{References}

1. Smith GE, Burrows $P$ (2008) Ultrasound diagnosis of fibroadenoma-is biopsy always necessary? Clin Radiol 63: 511-515.

2. Hamilton L, Evans A, Cornford E (2008) Ultrasound diagnosis of fibroadenoma-is biopsy always necessary? Clinical Radiology 63: 1070-1071.

3. Jayasinghe Y, Simmons PS (2009) Fibroadenomas in adolescence. Curr Opin Obstet Gynecol 21: 402-406.

4. El-Wakeel H, Umpleby HC (2003) Systematic review of fibroadenoma as a risk factor for breast cancer. Breast 12: 302307.

5. Hughes LE, Mansel RE, Webster DJ (1987) Aberrations of normal development and involution (ANDI): a new perspective on pathogenesis and nomenclature of benign breast disorders. Lancet 2: 1316-1319.

6. Turbey WJ, Buntain WL, Dudgeon DL (1975) The surgical management of pediatric breast masses. Pediatrics 56: 736-739.

7. Kuijper A, Mommers EC, van der Wall E, van Diest PJ (2001) Histopathology of fibroadenoma of the breast. Am J Clin Pathol 115: 736-742.

8. Stavros AT, Thickman D, Rapp CL, Dennis MA, Parker SH, et al. (1995) Solid breast nodules: use of sonography to distinguish between benign and malignant lesions. Radiology 196: 123-134.

9. Maxwell JR, Bugbee ME, Wellisch D, Shalmon A, Sayre $J$, et al. (2000) Imaging-guided core needle biopsy of the breast: Study of psychological outcomes. Breast J 6: 53-61.

10. Cant PJ, Madden MV, Close PM, Learmonth GM, Hacking EA, et al. (1987) Case for conservative management of selected fibro-adenomas of the breast. Br J Surg 74: 857-859.

11. Wilkinson S, Anderson TJ, Rifkind E, Chetty U, Forrest AP (1989) Fibroadenoma of the breast: a follow-up of conservative management. $\mathrm{Br} J$ Surg 76: 390-391.

12. Dixon JM, Dobie V, Lamb J, Walsh JS, Chetty U (1996) Assessment of the acceptability of conservative management of fibroadenoma of the breast. Br J Surg 83: 264-265.

13. Ozello L, Gump FE (1985) The management of patients with carcinomas in fibroadenomatous tumours of the breast. Surg Gynecol Obstet 160: 99-104.

14. Deschenes L, Jacob S, Fabia J, Christen A (1985) Beware of breast fibroadenomas in middle-aged women. Can $\mathrm{J}$ Surg 28: 372-374.

15. (2014) Breast cancer incidence (invasive) statistics. Cancer research UK.

16. Hughson AVM, Cooper AF, McArdle CS, Smith DC (1988) Psychosocial morbidity in patients awaiting breast biopsy. Journal of Psychosomatic Research 32: 173-180. 